\title{
Perfectly Secure Multiparty Computation and the Computational Overhead of Cryptography
}

\author{
Ivan Damgård $^{1}$, Yuval Ishai ${ }^{2, \star}$, and Mikkel Krøigaard ${ }^{3, \star \star}$ \\ 1 University of Aarhus, Denmark \\ ivan@cs.au.dk \\ 2 Technion and UCLA \\ yuvali@cs.technion.ac.il \\ 3 Eindhoven University of Technology \\ m.kroigaard@tue.nl
}

\begin{abstract}
We study the following two related questions:
- What are the minimal computational resources required for general secure multiparty computation in the presence of an honest majority?

- What are the minimal resources required for two-party primitives such as zero-knowledge proofs and general secure two-party computation?

We obtain a nearly tight answer to the first question by presenting a perfectly secure protocol which allows $n$ players to evaluate an arithmetic circuit of size $s$ by performing a total of $\mathcal{O}\left(s \log s \log ^{2} n\right)$ arithmetic operations, plus an additive term which depends (polynomially) on $n$ and the circuit depth, but only logarithmically on $s$. Thus, for typical largescale computations whose circuit width is much bigger than their depth and the number of players, the amortized overhead is just polylogarithmic in $n$ and $s$. The protocol provides perfect security with guaranteed output delivery in the presence of an active, adaptive adversary corrupting a $(1 / 3-\varepsilon)$ fraction of the players, for an arbitrary constant $\varepsilon>0$ and sufficiently large $n$. The best previous protocols in this setting could only offer computational security with a computational overhead of poly $(k, \log n, \log s)$, where $k$ is a computational security parameter, or perfect security with a computational overhead of $\mathcal{O}(n \log n)$.

We then apply the above result towards making progress on the second question. Concretely, under standard cryptographic assumptions, we obtain zero-knowledge proofs for circuit satisfiability with $2^{-k}$ soundness error in which the amortized computational overhead per gate is only polylogarithmic in $k$, improving over the $\omega(k)$ overhead of the best previous protocols. Under stronger cryptographic assumptions, we obtain similar results for general secure two-party computation.
\end{abstract}

\section{Introduction}

This work studies two different but closely related questions: the complexity of secure multiparty computation (MPC) in the presence of an honest majority,

\footnotetext{
* Supported by BSF grant 2008411, ISF grant 1310/06, and NSF grants 0830803, 0716835, 0627781 .

** Most of the work done at the University of Aarhus.
} 
and the complexity of two-party cryptographic primitives such as zero-knowledge proofs and secure two-party computation.

\subsection{The Complexity of MPC}

We consider the question of MPC over secure point-to-point channels in the presence of an active (malicious) adversary, who may corrupt up to some constant fraction $\delta$ of the $n$ players. In this work we focus on the case of an honest majority, where $\delta<1 / 2$. Unlike the case of MPC with no honest majority, in this case it is possible to guarantee output delivery and provide unconditional security. Following the initial feasibility results of [17/3/8 27], a long sequence of works, initiated by 14151910 , attempted to minimize the communication and computation resources required for general MPC in this setting.

To make the question cleaner and less sensitive to variations in the model, we adopt the following standard conventions. First, to measure the growth of complexity with the number of players, we consider $n$ as a parameter which tends to infinity. A large value of $n$ captures not only computations which combine inputs from many players, but also "cloud computing" scenarios in which a large number $n$ of untrusted or unreliable servers are used to distribute computations on inputs that originate from a small number of clients or even from just a single client. Second, to eliminate from consideration an additive overhead which depends (polynomially) on $n$ and a security parameten 11 but does not grow with the complexity of the functionality $f$, we assume the circuit complexity of $f$ to be much bigger than $n$. This is in line with most typical MPC application scenarios, and may capture both complex computations on small inputs and simple computations on massive inputs.

More concretely, we consider the task of securely evaluating a function $f$ represented by a boolean circuit $C$ whose inputs and outputs are arbitrarily partitioned between the $n$ players. We let $k$ denote a security parameter, such that the simulation error of the protocol is bounded by $2^{-k}$. (This should hold for computationally unbounded adversaries in the case of statistical security and for $2^{k}$-bounded adversaries in the case of computational security; the parameter $k$ can be ignored in the case of perfect security.) We say that a general MPC protocol has computational overhead $c(n, k, s)$ if for all positive integers $n, k, s$, and circuit $C$ of size $s$, the total number of bit operations 2 performed by all $n$ players together is at most $s \cdot c(n, k, s)+\operatorname{poly}(n, k, \log s)$. The computational overhead can be thought of as the amortized multiplicative price for achieving security: the ratio between the cost of securely distributing an expensive task between $n$ players and the cost of a centralized (insecure) solution for the same task.

Note that the computational overhead of a protocol implies a similar bound on its communication overhead with respect to the circuit size. However, in light

\footnotetext{
${ }^{1}$ Such an overhead is very sensitive to the underlying network and MPC model, and is required in our settings even for performing the simple MPC task of broadcasting a single bit.

${ }^{2}$ Our count of bit operations includes both local computations and point-to-point communication.
} 
of Gentry's recent candidate for a fully homomorphic encryption scheme [16], the circuit size should no longer be generally seen as a barrier for the communication complexity of MPC. This notion still looks meaningful in the setting of unconditional security or for circuits whose input or output length are comparable to their size. See Section 8 for further discussion.

The computation and communication overhead of the first general MPC protocols 1738 were large polynomials in $n, k$ (e.g., $\mathcal{O}\left(n^{8}\right)$ for a naive implementation of the perfectly secure BGW protocol over a point-to-point network [319]). Following a long sequence of works (see [13] for a survey) the current state of the art can be summarized as follows. For simplicity, we do not state the resilience level of each protocol. Using a general protocol composition technique from [6 18 13, all protocols can be made nearly optimally resilient with the same asymptotic overhead.

In the setting of computational security, an overhead of $c(n, k, s)=\operatorname{poly}(k$, $\log n, \log s)$ was achieved in 13 . This protocol can be realized with a constant number of rounds under standard cryptographic assumptions.

In the case of unconditional security, all efficient MPC protocols from the literature require the round complexity to grow with the circuit depth $d$. Since all players in these protocols are active in each round, we redefine computational overhead for the unconditional case to allow an additive term of $\operatorname{poly}(n, k, d, \log s)$ (the exponent of $d$ should be extremely low here, or the term can become dominant). The computational overhead of the best perfectly secure protocol prior to this work 2 was $n \cdot \operatorname{polylog}(n)$. This protocol has a similar communication overhead. In the case of statistical security and protocols which take inputs from and deliver outputs to only a constant number of clients (but still distribute the computation among $n$ servers) a variant of the protocol from [11] based on algebraic geometric secret-sharing [9] (see [20 22]) has computation overhead of $k \cdot \operatorname{polylog}(n)$ and communication overhead of $\mathcal{O}(1)$.

This state of the art leaves open several natural questions:

- Can the computational overhead be simultaneously sublinear in both $n$ and $k$ in any MPC model? This question turns out to be relevant for the applications discussed in Section 1.3 below.

- Can the computational overhead be sublinear in $n$ with perfect security, or alternatively with statistical security even when inputs can originate from all players (as opposed to a constant number of clients as in 1122])? These questions are open even for the easier case of communication overhead.

\subsection{Our Results}

We present a perfectly secure general MPC protocol whose computational overhead is polylogarithmic in $n$, answering the above questions affirmatively.

More concretely, the protocol can tolerate an active, adaptive adversary corrupting up to a $1 / 3-\varepsilon$ fraction 3 of the players, for an arbitrary constant

\footnotetext{
${ }^{3}$ In our model we assume that only point-to-point channels are available, in which case it is impossible to achieve unconditional security with guaranteed output delivery if at least $1 / 3$ of the players can be corrupted.
} 
$\varepsilon>0$ and all sufficiently large $n$. The computational (and communication) complexity required for evaluating a boolean circuit $C$ of size $s$ and depth $d$ is $\operatorname{poly} \log (n) \log s \cdot s+d^{2} \cdot \operatorname{poly}(n, \log s)$. If $C$ is an arithmetic circuit over a finite field of size bigger than $n$, the total computational work involves $\mathcal{O}\left(\log ^{2} n \log s\right.$. $s)+d^{2} \cdot \operatorname{poly}(n, \log s)$ arithmetic operations and the communication includes $\mathcal{O}(\log n \log s \cdot s)+d^{2} \cdot \operatorname{poly}(n, \log s)$ field elements.

Alternatively, in the case where $d^{2}$ is too large, we provide an option to increase the circuit size by a factor $\log d$ while decreasing the $d^{2}$ factor to $d \log d$. The intuition is that the first factor on the second term is $d X$, where $X$ is defined as follows. Dividing the circuit into layers in the natural way, we define the number $X$ to be the maximal number of layers reachable by one wire from any given layer. In general, $X=\mathcal{O}(d)$ and so the factor is $d^{2}$. With our alternative approach, $X=\mathcal{O}(\log d)$ and so the factor is $d \log d$. The real calculation is a bit more involved, but this is the basic idea.

Thus, with the above alternative, the computational complexity for an arithmetic circuit becomes $\mathcal{O}\left(\log ^{2} n \log s \log d \cdot s\right)+d \log d \cdot \operatorname{poly}(n, \log s)$, and similarly for the other complexities.

Since the modification of the circuit increases its size by a factor $\log d$, it is not always the best solution. Only for circuits with a large depth is the alternative a good choice. Furthermore, the $d^{2}$ factor is the result of a somewhat pessimistic worst-case analysis, and for most typical circuits the additive term grows only linearly with $d$.

As a final remark about our protocol, it seems "lean" enough to be implemented in practice. This should be contrasted with the previous best protocol from [13, which involves a distributed evaluation of a pseudorandom function for every gate in the circuit.

Techniques. Our protocol employs several techniques that were used in previous works along this line, including the share-packing technique from 14, allowing to secret-share a block of secrets with a low amortize cost, and the efficient verifiable secret sharing protocol from 213. The main technical challenge is to perform "non-homogenous" computations on pairs of blocks, i.e., ones that are different from coordinate-wise addition or multiplication of blocks. We address this challenge by embedding the computation in a special form of a universal circuit based on the so-called Beneš network [5129. The high level idea is that the structure of the circuit reduces the computation in a given layer of the circuit to an arbitrary permutation between blocks (which can be done locally), homogenous operations, and a logarithmic number of distinct permutations within blocks. We propose an efficient procedure for the latter. See Section 4 for a more detailed technical overview.

An independently interesting contribution is a new methodology for the security analysis of honest-majority MPC protocols. Similarly to most protocols of this type, our protocol is composed from subprotocols that generate auxiliary secret shared values to help in the computation, a subprotocol for sharing the inputs, and finally a "layer-protocol" that performs secure computation corresponding to one layer of the circuit, i.e., it starts with the shares of values going 
into the layer, consumes some auxiliary shared values, and outputs shares of values coming out of the layer. Our proof of security first proves all subprotocols to be UC secure. We then define a functionality $\mathcal{F}_{i}$ that takes inputs from the players and outputs shares of the values output by the $i$ 'th layer of the circuit (where layer 0 just produces the inputs to the circuit). We then show that $\mathcal{F}_{0}$ can be implemented by calling the auxiliary subprotocols, and $\mathcal{F}_{i}$ for $i>0$ can be (UC-)implemented by calling $\mathcal{F}_{i-1}$ and then executing the layer-protocol.

We believe this may be the first example of a general honest-majority MPC protocol with a fully modularized proof of security. The main challenge is that it is non-trivial to define functionalities for the subprotocols such that 1) the subprotocol actually realizes the functionality and 2) the functionality provides what is needed in the larger context. It is well known that even for a simple task such as digital signatures, defining the "right" functionality is not easy.

In our case, the main idea turn out to be that a functionality that is supposed to output shares of some secrets, should not simply choose those shares on its own and send them to the players, although that may seem like the most natural approach. Instead, our functionalities ask the adversary which shares it wants the corrupted players to get, and the functionality then chooses shares for the honest players conditioned on the shares obtained from the adversary and the secret. In a sense, this models the fact that we do not care about the distribution of shares the adversary sees, as long as the secret is safe.

\subsection{The Computational Overhead of Cryptography}

A somewhat unexpected motivation for this work comes from the recent applications of honest-majority MPC to two-party primitives such as zero-knowledge proofs and general secure two-party computation [20|22]. We note that these general tasks can be used as building blocks for more specialized two-party tasks such as identification or different flavors of signatures.

The computation and communication overhead of standard two-party cryptographic primitives can be defined similarly to the overhead of MPC as defined above, except that here $n$ is viewed as a constant and $s$ corresponds to work required for an insecure implementation (e.g., length of message in case of encryption, or size of witness verification circuit in the case of zero-knowledge). For instance, typical implementations of encryption have a constant communication overhead, but a poly $(k)$ computation overhead 4 In contrast, for typical implementations of zero-knowledge proofs or secure two-party computation protocols from the literature, both the communication and computation overhead are $\operatorname{poly}(k)$.

In 21] it was shown that, under plausible assumptions, various primitives including encryption, signatures, and secure two-party computation in the semihonest model can be implemented with a constant computational overhead. For

4 Since for the purpose of concreteness we consider attackers that run in time $2^{k}$, this requires to assume that the underlying hardness assumption is $2^{n^{\varepsilon}}$-strong for some $\varepsilon>0$. 
primitives such as encryption, commitment, hashing, and signatures, constructions with polylog $(k)$ overhead relying on lattice-based assumptions or errorcorrecting codes were given in 26241].

Obtaining similar results for zero-knowledge proofs and secure two-party computation against malicious parties is one of the main questions left open in [21]. Combining our main result with general transformations from 2022 , we can make progress on the this question. Concretely, under standard cryptographic assumptions (e.g., assuming $2^{n^{\varepsilon}}$-hardness of decoding random linear codes [1]), our main result yields zero-knowledge proofs for circuit satisfiability with $2^{-k}$ soundness error and simulation error, in which the amortized computational overhead per gate is only polylogarithmic in $k$, improving over the $\omega(k)$ overhead of the best previous protocols under any assumptions. Under stronger cryptographic assumptions, we obtain similar results for general secure two-party computation with simulation error $2^{-k}$. Both types of protocols are unconditionally secure when implemented in the natural hybrid model (i.e., using ideal commitments in the case of zero-knowledge, or oblivious transfer in the case of secure computation). This implies that all "cryptographic" computations can be done during a preprocessing stage, before the actual inputs are known. See Section 7 for more details.

\section{The Model}

We consider the standard setting of perfectly UC-secure MPC [7, with guaranteed output delivery, over a synchronous network of secure point-to-point channels. Our protocols also employ a broadcast primitive, but since the number of broadcasts will be small they can be simulated over point-to-point channels without affecting the amortized overhead.

The players in our protocol are divided into three categories: input clients who contribute inputs, output clients who receive outputs, and $n$ servers who help distribute the computation. To simplify the asymptotic complexity expressions, the number of clients is assumed to be $\mathcal{O}(n)$. Note that a player in the protocol is permitted to have one or more roles, and therefore this client-server model generalizes the usual model where every player has all three roles. The adversary is unbounded, active and adaptive, may corrupt up to $t$ servers and any number of clients, where $t$ is some constant fraction of $n$. (Concretely, one can use $t=n / 8$ in the basic version of our protocol.)

We assume that the functionality $f$ computed by the protocol is described by an arithmetic circuit $C$ over a finite field $\mathbb{Z}_{p}$, where $p>2 n$. (In the case of boolean circuits, we can use the least $p$ which satisfies this requirement. This results in an additional logarithmic communication overhead and polylogarithmic computation overhead.) The inputs and outputs of $C$ may be arbitrarily partitioned between the input clients and the outputs clients, respectively.

It will be convenient to partition the gates into layers, such that each layer gets its input only from the previous layers and provides output to subsequent layers. This can be done by partitioning the gates according to the length of a 
longest path from an input. The size of the circuit $C$ is written as $|C|$, and it is defined to be the number of gates plus the number of wires. Its depth is the length of the longest path from an input to an output, which is equal to the number of layers in the case of layered circuits.

Finally, since our efficiency goals are impossible to meet if each server needs to read an entire description of $C$, we separate the protocol compilation from the protocol execution. The protocol compiler takes a description of an arithmetic circuit $C$ (whose inputs and outputs are partitioned between the clients) and a number of servers $n$ and generates the "code" of each player in the protocol. When analyzing the complexity of the protocol we count only the cost of the protocol execution (combined over all players), but note that the protocol compilation can be performed with the same asymptotic computational cost as executing the protocol.

\section{Packed Secret-Sharing}

We will use the packed secret-sharing technique introduced by Franklin and Yung [14]. This is similar to standard Shamir secret-sharing [28] over $\mathbb{Z}_{p}$, but where a block of $l$ different values $\left(x_{1}, . ., x_{l}\right)$ are shared at once using a polynomial that evaluates to $x_{1}, \ldots, x_{l}$ in $l$ distinct points. For privacy if $t$ players are corrupted, the polynomial must be random of degree at most $d=t+l-1$. We need that, from a set of $n$ shares, one from each player, where at most $t$ are incorrect, the correct block of secrets can be efficiently determined, even if the polynomial has degree up to $2 d$. This will be the case if we set $t=n / 8$ and $l=n / 4$. Also, to have enough distinct evaluation points, we need that $p>2 n$. This is the same variant of packed secret sharing as was used in [13], which we refer to for further details.

Denote by $[x]_{d}$ a packed secret-sharing of the block $x$ using a polynomial of degree at most $d$. Any vector of shares $\left\{s_{1}, \ldots, s_{n}\right\}$ among $n$ servers is called $d$-consistent if the shares correctly match a degree at most $d$ polynomial in the $n$ first points and therefore uniquely defines a block of secrets.

Throughout the paper we will need many different protocols dealing with block sharings. Most notably we need verifiable secret-sharing for the input and reconstruction with error correction for the output. In Section 5 on page 453 we describe the known protocols that we will use.

\section{Overview of the Protocol}

Using packed secret sharing, it is straightforward to do secure addition or multiplication on $l$ values in parallel, at the price of what a single operation would cost using normal secret sharing. This was already observed in 14 and can be used to compute the circuit $C$ securely and efficiently if we arrange it such that every layer contains only one type of gates, and if we can produce sets of shared blocks $S_{1}, S_{2},$. such that blocks in $S_{i}$ contain the $i$ 'th input bit to the gates in a given layer, in some fixed order. We will call this a correct line-up for the given layer. 
Demanding correct line-up is a problem, however: It implies that the values in the computation will have to be permuted between layers in arbitrary ways that depend on the concrete circuit. This is not easy to implement efficiently using packed secret sharing. We solve this problem by first constructing from $C$ a new circuit $C^{\prime}$ that computes the same function but is more well-behaved. More precisely, we have

Lemma 1. Given an arithmetic circuit $C$ that is at least $l$ gates wide, there is an efficient algorithm to transform it into another circuit $C^{\prime}$ with the following properties:

1. $C^{\prime}(x)=C(x)$ for all inputs $x$.

2. Every layer contains only one type of gate.

3. If all values are stored in blocks using packed secret sharing where the block size $l$ is a 2-power, the action between any two layers to achieve correct lineup is to permute the blocks and then in some blocks permute the elements within the block, where the same permutation applies to all blocks in the layer 5 . In the entire circuit, only $\log l$ different permutations are needed to handle permutations within blocks.

4. $\left|C^{\prime}\right|=\mathcal{O}\left(|C| \log |C|+\operatorname{depth}(C)^{2} n \log ^{3}|C|\right), \operatorname{depth}\left(C^{\prime}\right)=\mathcal{O}\left(\log ^{2}|C| \operatorname{depth}(C)\right)$.

The restriction on the width of the circuit is fairly insignificant, since $n$ is generally small compared to the circuit size. Some of the layers in $C^{\prime}$ will not be a block wide, but since those layers also do not require a permutation, it will cause no problems.

We show in the full version (available on ePrint) [12 how this construction works in detail. The basic idea is to handle the arbitrary permutations needed in $C$ by inserting a small piece of circuitry that permutes the values as desired. This subcircuit can be made very regular using permutation networks as described by Waksman [29]. These are based on Beneš networks [5]. It follows from the construction that $C^{\prime}$ only contains addition, multiplication and H-gates, where $\mathrm{H}$ swaps two input values $x, y$ or leaves them alone, depending on a control-bit c: $\mathrm{H}(x, y, c)=(c x+(1-c) y, c y+(1-c) x)$.

Now, given the input arithmetic circuit $C$, we first transform it into $C^{\prime}$ as described in the lemma. We begin our actual computation by secret-sharing the input values in blocks of size $l=\Theta(n)$, where $l$ is a 2-power, and we then go through $C^{\prime}$ layer by layer, computing at each stage the output values from the layer in packed secret-shared form. Once we have the output from the last layer, shares of these are sent to the output clients for reconstruction.

Going into each layer we permute the shared blocks we have so far as needed to get correct line-up for the layer, and then do the computation required. The only non-trivial issue is how to permute the elements inside a shared block, i.e., how to compute $[\pi(x)]_{d}$ from $[x]_{d}$ for a permutation $\pi$. The idea is to first precompute pairs of the form $[r]_{d},[\pi(r)]_{d}$ for random blocks $r$. We show below how to generate many such pairs using the same $\pi$ at a small amortized cost per

\footnotetext{
${ }^{5}$ In some cases, it may additionally be necessary to discard some blocks.
} 
pair. This is sufficient, since by the above lemma, we only need a small number of different permutations. The idea then is to reveal $x+r$ to a single server, who then locally computes $\pi(x+r)$ and secret-shares it, proving in the process that $[\pi(x+r)]_{d}$ was correctly formed. This can be done efficiently if we do many blocks in parallel. Then, given $[\pi(x+r)]_{d}=[\pi(x)+\pi(r)]_{d}$ and $[\pi(r)]_{d}$, players subtract shares locally to get $[\pi(x)]_{d}$.

\section{Subprotocols}

In the previous sections, we have covered how to evaluate a circuit $C$ by transforming it into $C^{\prime}$ and computing layer by layer. We begin this section by listing known protocols that we will be using for this. Subsequently we cover new protocols we propose.

Known protocols. From [13] we borrow the following protocols:

- Share $(D, d)$ : A dealer $D$ computes shares of a block of $l$ secrets using a degree $d$ polynomial and sends a share to each player. Communication is $\mathcal{O}(n)$ and computation is $\mathcal{O}(n \log n)$.

- $\operatorname{Reco}(R, d)$ : Assumes a block has been shared using a polynomial of degree at most $d$. All players send their shares of the block to $R$, who uses standard error correction techniques to reconstruct the block. Communication is $\mathcal{O}(n)$ and computation is $\mathcal{O}(n \log n)$.

- RobustShare $(d)$ : This protocol basically implements verifiable secret-sharing for one or more dealers who want to secret-share $\Theta(n)$ blocks each using polynomials of degree $d$. The functionality it implements, $\mathcal{F}_{\text {RobustShare }}$, is shown in Figure 1 on the next page.

- RanDouSha $(d)$ : Generates a vector of random blocks and a degree $d$ and a degree $2 d$ sharing of each block. More precisely, it implements the functionality shown in Figure 2 on the following page.

- RobustReshare $\left(d, d^{\prime}\right)$ : Takes as input a number of secret shared blocks. For each input $[x]_{d}$ it outputs a new sharing $[x]_{d^{\prime}}$. However, it does not keep $x$ secret.

- SemiRobustShare $(d)$ : Same as RobustShare $(d)$, but the adversary can cause some of the honest dealers to fail. However, during the entire global protocol, he can only make up to $t$ honest dealers fail.

For every protocol above except for the first two, the communication complexity is $\mathcal{O}\left(\beta n^{2}\right)$, and the computational complexity is $\mathcal{O}\left(\beta n^{2} \log n\right)$, for handling $\beta$ groups of $\Theta(n)$ blocks. In both cases we must additionally pay $\mathcal{O}\left(n^{2}\right)$ per complaint. Complaints are handled as in our protocol RandomPairs in Figure 4 on page 456 . Since each complaint results in at least one corrupted player being eliminated from the protocol, at most $t$ complaints can occur in total.

Furthermore, there is a minimal cost for these protocols, since they are built to handle groups of blocks and not just single blocks at a time. RobustShare for example always costs at least as much as for $\beta=n$. For a protocol like 
1. Receive from all honest players the identities of the dealers and the number of blocks they want to share. Abort if the input is inconsistent. Receive also a set of input blocks to share from each honest dealer.

2. Send "Shares?" to the adversary together with the identities of the dealers and the number of blocks they want to share.

3. Receive from the adversary, for each block to be shared by an honest dealer, one share for each corrupted player (this should be thought of as the shares the adversary wants the corrupted players to receive). For each corrupt dealer, receive a polynomial of degree at most $d$.

4. For each block to be shared by an honest dealer, choose a random polynomial of degree at most $d$ that is consistent with the block and the shares the adversary chose for the corrupted players. Compute and send the resulting shares to the honest players, and send the entire polynomial to the dealer.

5. For each block to be shared by a corrupt dealer, if the adversary sent a polynomial of correct degree, compute shares using this polynomial and send them to the players, otherwise tell all players that the dealer failed.

Fig. 1. The functionality $\mathcal{F}_{\text {Robust Share }}$

SemiRobustShare, it is possible to handle $\beta=1$ efficiently, but then we need to add $\mathcal{O}\left(n^{3}\right)$ for $n$ broadcasts. However, as we will show later, these cases make no difference in our final complexity; for this we do not care about how well our protocols handle a small number of elements, we care about how they scale.

In [13] there is a proof of perfect privacy and correctness for each of the protocols above, but it was not proved there that RanDouSha and Robust Share implement the corresponding functionalities. A proof of this follows quite easily from correctness and privacy in the same way as in the proof for the protocol RandomPairs, which we present in detail below.

We define functionalities only for some of the protocols above. The rest are mentioned because we use them as parts of other protocols. The final UC proof in the full version [12] only requires these parts to have perfect privacy and correctness.

\subsection{Permuting Elements within a Block}

The basic idea behind our protocols for permuting the set of elements within each block for a vector of blocks was already explained in Section 4 . To use this idea, we need to be able to produce pairs of sharings $[r]_{d},[\pi(r)]_{d}$ for random $r$ 's, and a server needs to be able to secret-share blocks while showing that they were correctly permuted. First we present the protocol RandomPairs for producing the required permuted pairs. The protocol for resharing and proving is simpler and yet very similar, and for that case we provide only a sketch. The protocol makes use of hyperinvertible matrices. A matrix is hyperinvertible if any intersection

1. Each honest player sends a natural number $r$ to $\mathcal{F}_{\text {double. }}$ If the honest players sent different values for $r, \mathcal{F}_{\text {double }}$ halts and outputs ABORT. Otherwise, send $r$ and message "Shares?" to the adversary.

2. The adversary chooses $2 r$ sets of shares for the corrupted players.

3. $\mathcal{F}_{\text {double }}$ chooses $r$ random blocks $\left(x_{1}, \ldots, x_{r}\right)$ and creates random sharings $\left(\left[x_{1}\right]_{d}, \ldots,\left[x_{r}\right]_{d}\right)$ and $\left(\left[x_{1}\right]_{2 d}, \ldots,\left[x_{r}\right]_{2 d}\right)$ such that they are consistent with the shares submitted by the adversary.

4. $\mathcal{F}_{\text {double }}$ outputs the resulting shares to the players.

Fig. 2. The functionality $\mathcal{F}_{\text {double }}$ 
between $k$ rows and $k$ columns of the matrix is invertible. In [2], it is described how such a matrix can be constructed. We refer to [2] for the details, but it is important to note, as was also done in [13, that we may use the $\mathcal{O}(n \log n) \mathrm{FFT}$ algorithms to multiply our hyperinvertible matrices onto vectors.

Creating Permuted Pairs. The functionality $\mathcal{F}_{\text {pairs }}$ shown in Figure 3 details our requirements for the creation of permuted pairs. It works almost exactly like $\mathcal{F}_{\text {double. }}$

1. Each honest player sends a natural number $r$ and a permutation $\pi$ to $\mathcal{F}_{\text {pairs }}$. If the honest players sent different values for $r$ or $\pi, \mathcal{F}_{\text {pairs }}$ halts and outputs ABORT. Otherwise, send $r$ and message "Shares?" to the adversary

2. The adversary chooses $2 r$ sets of shares for the corrupted players.

3. $\mathcal{F}_{\text {pairs }}$ chooses $r$ random blocks $\left(x_{1}, \ldots, x_{r}\right)$ and chooses random sharings $\left(\left[x_{1}\right], \ldots,\left[x_{r}\right]\right)$ and $\left(\left[\pi\left(x_{1}\right)\right], \ldots,\left[\pi\left(x_{r}\right)\right]\right)$ such that they are consistent with the shares submitted by the adversary.

4. $\mathcal{F}_{\text {pairs }}$ outputs the chosen shares to the players.

Fig. 3. The functionality $\mathcal{F}_{\text {pairs }}$

An observation is needed before we present the protocol. Say we have some permutation $\pi$ on $l$ different elements, a vector of random blocks $\left(x_{1}, \ldots, x_{n}\right)$, and a vector of $y_{i}=\pi\left(x_{i}\right)$. Now suppose we apply some $m$ by $n$ matrix $M$ and get the resulting vectors $\left(x_{1}^{\prime}, \ldots, x_{m}^{\prime}\right)$ and $\left(y_{1}^{\prime}, \ldots, y_{m}^{\prime}\right)$.

Applying $M$ to a vector of blocks corresponds to applying $M$ to $l$ different vectors at once. Permuting all blocks and then applying $M$ clearly has the same result as applying $M$ and then permuting the resulting blocks. More precisely, after applying $M, \pi\left(x_{i}^{\prime}\right)=y_{i}^{\prime}$.

We now present the protocol RandomPairs. It is run in parallel for all of the players with the restriction that $n-3 t=\Omega(n)$. The matrix $M$ is hyperinvertible of dimension $n$ by $n-2 t$, and $X$ is hyperinvertible of dimension $n-2 t$ by $n-2 t$. The protocol is shown in Figure 4 on the next page.

Proposition 1. The protocol RandomPairs securely realizes $\mathcal{F}_{\text {pairs }}$ in the $U C$ model with perfect security against an active and adaptive adversary corrupting at most $t$ players, where $n-3 t=\Omega(n)$. RandomPairs creates $\Theta\left(n^{2}\right)$ permuted pairs at a time with a communication complexity of $\mathcal{O}\left(n^{3}\right)$, and a computational complexity of $\mathcal{O}\left(n^{3} \log n\right)$. In both cases, we add $\mathcal{O}\left(n^{2}\right)$ per complaint.

Proof. The proof is divided into three parts. The first two are correctness and simulation, and together they prove security in the UC model. The last part deals with the complexity.

Correctness: To show correctness, we must prove that all generated pairs are consistently shared and correctly permuted. Consider the set of players $\mathcal{P}$. If we denote by $\mathcal{P}^{\prime}$ the subset of non-eliminated players, we know that by the end of the elimination step, only sharings coming from players in $\mathcal{P}^{\prime}$ will be used.

We know that for any dealer $D \in \mathcal{P}^{\prime}$, there are no conflicts $\left\{P_{i}, D\right\} \in \mathcal{C}$ for any $P_{i} \in \mathcal{P}^{\prime}$. If there were such conflicts, they would have caused the elimination of either $D$ or $P_{i}$ in the elimination phase. This means that all honest players in 
1. Sharing

For each player $D$ acting as dealer, and each group $g$ of pairs to make, run the following in parallel:

(a) $D$ picks random blocks $\left(x_{1}, \ldots, x_{n-2 t}\right)$ and $\left(y_{1}, \ldots, y_{n-2 t}\right)=\left(\pi\left(x_{1}\right), \ldots, \pi\left(x_{n-2 t}\right)\right)$.

(b) $D$ shares the $x_{i}$ and the $y_{i}$ using protocol Share.

(c) All players calculate

$$
\begin{aligned}
& \left(\left[x_{1}^{\prime}\right], \ldots,\left[x_{n}^{\prime}\right]\right)=M\left(\left[x_{1}\right], \ldots,\left[x_{n-2 t}\right]\right) \\
& \left(\left[y_{1}^{\prime}\right], \ldots,\left[y_{n}^{\prime}\right]\right)=M\left(\left[y_{1}\right], \ldots,\left[y_{n-2 t}\right]\right) .
\end{aligned}
$$

(d) For all $i$, all players $P_{j}$ send their shares of $\left[x_{i}^{\prime}\right]$ and $\left[y_{i}^{\prime}\right]$ to $P_{i}$.

(e) For all $i$, the dealer $D$ sends all shares of $\left[x_{i}^{\prime}\right]$ and $\left[y_{i}^{\prime}\right]$ to $P_{i}$.

2. Checking

Initialize $\mathcal{C}=\emptyset$. This set will contain sets of conflicting players. Now for each player $P_{i}$ in parallel:

(a) $P_{i}$ checks that the sharings received for $x_{i}^{\prime}$ and $y_{i}^{\prime}$ by all $D$ for all groups are consistent, and that $y_{i}^{\prime}=\pi\left(x_{i}^{\prime}\right)$. For any pair $\left(P_{j}, D\right)$ where this check went well, $P_{i}$ also checks that he received the same shares from all pairs of dealers $D$ and $P_{j}$. If all goes well, he broadcasts a 1 , and a 0 is broadcast if one or more checks fail.

(b) If $P_{i}$ broadcast a 0 , he now proceeds to broadcast the number of complaints he intends to make. The complaints are then handled as described in the following. If at any point $P_{i}$ broadcasts badly formatted complaints or the same complaint more than once, $P_{i}$ is immediately eliminated and ignored.

(c) If a dealer $D$ dealt inconsistent shares or the pairs were not correctly permuted, $P_{i}$ broadcasts (CONFLict, $P_{i}, D$ ). All players include the set $\left\{P_{i}, D\right\}$ in $\mathcal{C}$.

(d) Otherwise, if $P_{i}$ sees that it has received different shares from some $P_{j}$ and $D$ for a group $g$, it broadcasts (CONFLICT, $D, P_{j}$, g $_{\text {, }}$ are $_{D}$, share $_{P_{j}}, w$ ), where $w$ indicates whether it is a conflict with shares of $\left[x_{i}^{\prime}\right]$ or $\left[y_{i}^{\prime}\right]$. Such conflicts are sent out for any relevant cases, but at most one conflict is sent out for any specific pair $\left(D, P_{j}\right)$.

i. If $D$ finds that share $_{D}$ does not match what he sent to $P_{i}$, he broadcasts (CONFLICT, $D, P_{i}$ ), and it is recorded in $\mathcal{C}$.

ii. If $P_{j}$ finds that share $_{P_{j}}$ does not match what he sent to $P_{i}$, he broadcasts (CONFLiCT, $P_{j}, P_{i}$ ). This is recorded in $\mathcal{C}$.

iii. If neither $D$ nor $P_{j}$ broadcasts a conflict, the conflicting set $\left\{D, P_{j}\right\}$ is included in $\mathcal{C}$.

\section{Elimination}

All players now locally run the following elimination algorithm:

(a) If there is a pair $\left\{P_{i}, P_{j}\right\} \in \mathcal{C}$ such that neither player has been eliminated so far, eliminate both players by removing them from the set $\mathcal{S}$ of player.

(b) Keep all pairs $\left(\left[x_{i}\right],\left[y_{i}\right]\right)$ shared by non-eliminated players, throw away the rest.

4. Postprocessing phase

(a) Reorder the players such that 1 through $n-2 t$ are non-eliminated.

(b) $\left(x_{i}^{j}, y_{i}^{j}\right)$ is the $i$ 'th pair of blocks known to the $j$ 'th player, for all non-eliminated $j$, and for each group.

(c) Every player calculates

$$
\begin{aligned}
\left(\left[a_{i}^{1}\right], \ldots,\left[a_{i}^{n-2 t}\right]\right) & =X^{-1}\left(\left[x_{i}^{1}\right], \ldots,\left[x_{i}^{n-2 t}\right]\right) \\
\left(\left[b_{i}^{1}\right], \ldots,\left[b_{i}^{n-2 t}\right]\right) & =X^{-1}\left(\left[y_{i}^{1}\right], \ldots,\left[y_{i}^{n-2 t}\right]\right) .
\end{aligned}
$$

for all $i \in\{1, \ldots, n-3 t\}$, and for each group.

(d) For each group, the output is given by the pairs $\left(\left[a_{i}^{j}\right],\left[b_{i}^{j}\right]\right)$ for $i, j \in\{1, \ldots, n-3 t\}$.

\section{Fig. 4. Protocol RandomPairs}

$\mathcal{P}^{\prime}$ agree that the shares they have received from dealers $D \in \mathcal{P}^{\prime}$ are consistent and represent correctly permuted pairs, and furthermore these shares agree with all shares received from $P_{j} \in \mathcal{P}^{\prime}$.

Now consider all non-eliminated honest players. We know that at least for every two players eliminated, one of the players must have been corrupted. Therefore, we have at least $n-2 t$ honest players in $\mathcal{P}^{\prime}$. Now select exactly $n-2 t$ of those and form the set $H$. It can be seen then that 


$$
\left(\left[x_{i}^{\prime}\right]\right)_{P_{i} \in H}=M_{H}\left(\left[x_{i}\right]\right)_{1 \leq i \leq n-2 t},
$$

where $M_{H}$ is a matrix containing only the rows of $M$ with indices corresponding to the players in $H$. Since $M_{H}$ is a square submatrix of a hyperinvertible matrix, it must be invertible. This means that

$$
\left(\left[x_{i}\right]\right)_{1 \leq i \leq n-2 t}=M_{H}^{-1}\left(\left[x_{i}^{\prime}\right]\right)_{P_{i} \in H} .
$$

The calculations above also hold for the $y_{i}$. We know that all pairs $\left(x_{i}^{\prime}, y_{i}^{\prime}\right)$ where $P_{i} \in H$ are guaranteed to be consistently shared and correctly permuted. Applying the linear transformation $M_{H}^{-1}$ preserves this property, and so we know that all of the original pairs $\left(x_{i}, y_{i}\right)$ must be correct as long as the dealer is in $\mathcal{P}^{\prime}$, but these are exactly the pairs we keep after the elimination phase.

Following the elimination phase, new pairs are created by applying yet another linear transformation. As before, linear transformations preserve the consistency of sharings and the property that pairs are correctly permuted, and thus correctness is ensured.

Simulation: To prove UC security, we must also show that we can construct a simulator $\mathcal{S}$ such that any environment $\mathcal{Z}$ cannot distinguish between the real world where it communicates with the adversary $\mathcal{A}$ and the ideal world where it communicates with $\mathcal{S}$. We do this by first proving perfect privacy (i.e. we prove that the adversary's view is independent of the secrets shared), and then we show how to use this and correctness to build a simulator.

For perfect privacy, all values seen by the adversary should be independent of the secret, which in this case is the set of output pairs. Throughout the protocol, $\mathcal{A}$ learns openings of sharings from honest players, and it knows its own sharings as well. It is these values that should be independent of the output. More specifically, we need only examine sharings by non-eliminated players, since the others are not used to create the output.

First, we prove that the sharings distributed by non-eliminated honest players are independent of the sharings opened towards $\mathcal{A}$. For any honest dealer and any group, let $I=\{1, \ldots, n-3 t\}$ be the indices of the initial blocks and $R$ those of the remaining blocks. Now choose a set $C$ of size $t$ that contains all indices of the corrupted players. The corrupted players now know openings of

$$
\left(\left[x_{i}^{\prime}\right]\right)_{i \in C}=M_{C}^{I}\left(\left[x_{i}\right]\right)_{i \in I}+M_{C}^{R}\left(\left[x_{i}\right]\right)_{i \in R},
$$

where $M_{A}^{B}$ means the matrix $M$ restricted to rows in $A$ and columns in $B$. A similar equation holds for the $y_{i}^{\prime}$. Since $|C|=|R|$, there is exactly one choice of blocks in $R$ that matches what the adversary can see for any set of blocks in $I$. In other words, the blocks opened to $\mathcal{A}$ are independent of the ones dealt by the honest dealers.

The final output blocks are created using the sharings from all non-eliminated servers, possibly including some corrupted servers. Therefore, we must also prove that the final outputs are independent of sharings from non-eliminated corrupt players. For the $a_{i}^{j}$ and any group (the proof is the same for the $b_{i}^{j}$ ), let $I=$ 
$\{1, \ldots, n-3 t\}$ be the set of the initial $n-3 t$ indices, $R$ the subsequent $t$, and $C$ a set of size $t$ containing the indices of all non-eliminated corrupted players (fill the rest of $C$ with other players if there are less than $t$ ). The adversary knows $x_{i}^{j}$ for all $j \in R$, so the sharings known to $\mathcal{A}$ are

$$
\left(\left[x_{i}^{j}\right]\right)_{j \in C}=X_{C}^{I}\left(\left[a_{i}^{j}\right]\right)_{j \in I}+X_{C}^{R}\left(\left[a_{i}^{j}\right]\right)_{j \in R},
$$

for all $i$. Since $|C|=|R|$, and since $X$ is hyperinvertible, $X_{C}^{R}$ is invertible. Therefore, for any set of blocks known to the adversary, there is exactly one choice of blocks $\left[a_{i}^{j}\right]_{j \in R}$ not output for any set of output blocks. In other words, the blocks dealt by $\mathcal{A}$ are independent of the output blocks. This concludes our proof of privacy.

We can now show how to construct a simulator $\mathcal{S}$. It simply runs dummy versions of the honest players and lets the execute the protocol with $\mathcal{A}$. We know that any values seen by $\mathcal{A}$ during the protocol are independent of the actual secrets shared, so the values generated by $\mathcal{S}$ towards $\mathcal{A}$ must be correctly distributed. When the protocol is done, the shares for corrupted players generated by the simulated run is fed into $\mathcal{F}_{\text {pairs }}$. The functionality now chooses the output sharing so to match these values, i.e. the honest players obtain shares that are consistent with a set of correctly distributed secrets and with the shares held by the adversary. By correctness of the protocol, this matches exactly the distribution of the output of a real protocol run.

The very last part of the proof is to deal with adaptive corruptions. First of all, if an honest player is corrupted during the protocol run but before we receive outputs from $\mathcal{F}_{\text {pairs }}$, we may simply open up one of the dummy parties to the adversary and continue from there. The only difficult part is if a server is corrupted after the output sharings have been chosen, because in that case the view of a dummy party does not match the output sharings. To adjust the view of a dummy party to the actual output shares of $\mathcal{F}_{\text {pairs }}$, we examine how these shares are constructed. We start by adjusting the shares of the $\left[a_{i}^{j}\right]$ for $j \in I$ (all of the following works in the same way for the $b_{i}^{j}$ ). The adversary knows the full sharings of

$$
\left(\left[x_{i}^{j}\right]\right)_{j \in C}=X_{C}^{I}\left(\left[a_{i}^{j}\right]\right)_{j \in I}+X_{C}^{R}\left(\left[a_{i}^{j}\right]\right)_{j \in R},
$$

so for those we simply pick the correct shares of $\left[a_{i}^{j}\right]$ for $j \in R$ to match the adjusted shares for $j \in I$. Now calculate $\left(\left[x_{i}^{j}\right]\right)_{j}=X\left(\left[a_{i}^{j}\right]\right)_{j}$ to find the remaining shares owned by the newly corrupted player. This of course means that the other dummy parties have to adjust their sharings from this point. The last problem is $x_{i}^{j}$ created by this player. We can easily adjust its sharing of those values to match what we need, but it also needs to match the values opened to the adversary during the sharing of them. Luckily, we already know that this is simply a matter of adjusting the randomness used in the sharing.

Complexity: We now examine the complexity of the protocol. Going through each step of the protocol and remembering that every server is a dealer, we see that each step has a maximum communication complexity of $\mathcal{O}\left(n^{3}\right)$. Clearly 
this is also the total communication complexity. The computational complexity is $\mathcal{O}\left(n^{3} \log n\right)$ plus the cost of each complaint, since in the slowest step, every server must check the consistency of $\Theta(n)$ sharings by interpolation, which can be done by using $\mathcal{O}(n \log n)$ FFT. Every complaint adds $\mathcal{O}\left(n^{2}\right)$ to both complexities for the broadcast.

Permuting Elements within Blocks. The next subprotocol PermuteWithinBlocks, and it is shown in Figure 5 takes as input the shares of blocks $\left(\left[x_{1}\right], \ldots,\left[x_{n}\right]\right)$, a vector of random pairs $\left(\left(\left[s_{1}\right],\left[\pi\left(s_{1}\right)\right]\right), \ldots,\left(\left[s_{n}\right],\left[\pi\left(s_{n}\right)\right]\right)\right)$, and the permutation $\pi$. It outputs shares of new sharings $\left(\left[\pi\left(x_{1}\right)\right], \ldots,\left[\pi\left(x_{n}\right)\right]\right)$. For this protocol, we prove correctness and privacy here, and use these properties in the simulation proof for the main protocol.

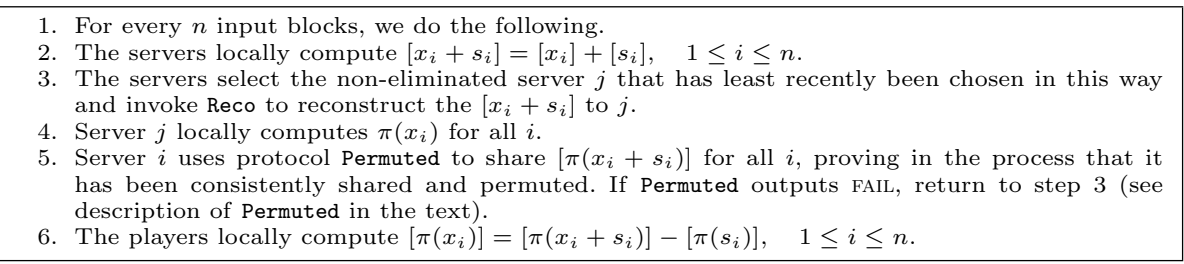

Fig. 5. Protocol PermuteWithinBlocks

Note that we only run the protocol for $n$ blocks at a time to limit the cost of Permuted failing. For efficiency, we must work on at least $n$ blocks at a time, so this is the natural choice. The protocol Permuted that was mentioned above is an adaptation of RandomPairs: there is only one dealer, server $j$. Rather than sharing both the $x_{i}$ 's and $\pi\left(x_{i}\right)$ 's, the server shares only $\pi\left(x_{i}\right)$, since servers already have shares of the $x_{i}$ 's in question. However some extra random $x_{i}$ 's are added to ensure privacy (recall that RandomPairs requires extra random blocks that will not be output). Otherwise, we do exactly the same as in RandomPairs but if FAIL if server $j$ is eliminated we stop immediately and output fail. The postprocessing phase is omitted, since there is only a single dealer who is allowed to know the (masked) secret.

It is perfectly private and correct by for the same reason that PermutedPairs is. As for the complexities, we consider permuting $\beta$ groups of $\Theta(n)$ blocks (i.e. we permute $\Theta(\beta n)$ blocks $)$. Ignoring broadcasts for a moment, we see that communication is at its most expensive when initially sharing, which $\operatorname{costs} \mathcal{O}\left(\beta n^{2}\right)$. The most expensive computational step is still checking, which $\operatorname{costs} \mathcal{O}\left(\beta n^{2} \log n\right)$. For both computation and communication, we need to add $\mathcal{O}\left(n^{3}\right)$ in broadcast costs in both cases (regardless of the number of groups) and a further $\mathcal{O}\left(n^{2}\right)$ per complaint.

For the protocol PermuteWithinBlocks, it is clear that we still have privacy, since random blocks are added before opening. Correctness is trivial from the construction. As for the complexities, the most expensive step is Permuted. So both computational and communication complexities are as above, with the 
exception that the cost is multiplied by the number of times we fail and have to rerun Permuted. Since each failure results in at least one corrupt player being eliminated, the worst case is having to rerun $t$ times.

\subsection{Multiplications}

As explained earlier, our circuit consists of only addition, multiplication and $\mathrm{H}-$ gates, where $\mathrm{H}(x, y, c)=(c x+(1-c) y, c y+(1-c) x)$. Since addition is trivially done by local computation, it is sufficient to explain how to handle multiplications. In order to do this, we need the protocol RobustReshare; as mentioned above it coverts a vector of blocks from being shared with degree $d_{1}$ to shares with degree $d_{2}$. In a nutshell, it publicly reconstructs the values and then reshares them. Assume that we are given shared blocks $[x]_{d},[y]_{d}$ with degree $d$ and sharings $[r]_{d},[r]_{2 d}$ of the same $r$ but with degree $d$ and $2 d$. The protocol Multiply then works as shown in Figure 6.

1. For every pair of blocks $x, y$ to multiply, we assume sharings $[r]_{d},[r]_{2 d}$ are available. The servers locally compute $[x y+r]_{2 d}=[x]_{d}[y]_{d}+[r]_{2 d}$.

2. RobustReshare is run to obtain $[x y+r]_{d}$ for all $x, y$

3. For every $x, y$ the servers locally compute $[x y]_{d}=[x y+r]_{d}-[r]_{d}$.

Fig. 6. Protocol Multiply

The pairs $[r]_{d},[r]_{2 d}$ we need can be generated using RanDouSha mentioned above. Correctness follows from correctness of RobustReshare. Privacy follows from privacy of RanDouSha since we can then assume the $r$ is uniformly random from the adversary's point of view. The complexity is clearly dominated by RobustReshare whose complexity was covered earlier.

\section{The Main Protocol}

The final protocol is described in Figure 8 on the facing page, while the functionality realized is in Figure 7 . This leads to:

1. The input clients send their inputs $\left(x_{1}, \ldots, x_{r}\right)$ to $\mathcal{F}_{C}$

2. $F_{C}$ distributes $\left(y_{1}, \ldots, y_{t}\right)=C\left(x_{1}, \ldots, x_{r}\right)$ to the intended output clients.

Fig. 7. The functionality $\mathcal{F}_{C}$ for the circuit $C$

Theorem 1. There exists $0<\delta<1 / 3$ such that given $n$ servers and an arithmetic circuit $C$ that is at least $\Omega(n)$ gates wide, the protocol EvalCircuit realizes $\mathcal{F}_{C}$ with perfect security in the $U C$ model against an active and adaptive adversary corrupting up to $t<\delta n$ servers.

The total communication complexity is

$$
\mathcal{O}(\log n \log |C| \cdot|C|)+\operatorname{poly}(n, \log |C|) \cdot \operatorname{depth}(C)^{2},
$$


Preprocessing: Transform $C$ into $C^{\prime}$.
Step 0: Input clients invoke the functionality $\mathcal{F}_{\text {Robustshare }}$ to share their inputs to the servers.
The servers invoke $\mathcal{F}_{\text {pairs }}$ and $\mathcal{F}_{\text {double }}$ to create a set $P_{i}$ of pairs and a set $D S_{i}$ of double
sharings for every layer $1 \leq i \leq d$ of $C^{\prime}$, where $d=\operatorname{depth}\left(C^{\prime}\right)$.
Step $i$ : For $1 \leq i \leq d$, we have from the previous layers the set $I_{i}$ of inputs for this layer as well as
pairs and double sharings $P_{i}$ and $D S_{i}$ for this layer. Layer $i$ is evaluated on $I_{i}$ by the servers
through local computations and a constant number of calls to Multiply.
The outputs of the layer may need to be permuted. If the blocks are to be permuted, they are
permuted by local computation. If the elements within the blocks need to be permuted, the
servers invoke PermuteWithinBlocks on the blocks in question.
Step $d+1$ : The servers open sharings to the relevant output clients using Reco.

Fig. 8. Protocol EvalCircuit

while the total computational complexity is

$$
\mathcal{O}\left(\log ^{2} n \log |C| \cdot|C|\right)+\operatorname{poly}(n, \log |C|) \cdot \operatorname{depth}(C)^{2} .
$$

The actual threshold in Theorem 1 on the facing page is quite far from the optimal $n / 3$ bound. To improve on this, we may use the player virtualization technique by Bracha [6] in the same way it was used in [13, to which we refer for the details of the construction. The basic idea is to construct virtual servers that run our protocol. To simulate each virtual server, a subset of the servers run a less efficient protocol, the inner protocol, that has a high threshold.

The difference from [13 is that here we are interested in perfect security. Therefore we need an inner protocol that also has perfect security. To this end, we can employ the BGW protocol [3]. Since it has threshold $n / 3$, the construction from [13] gives us a threshold of $n / 3-\varepsilon$ for sufficiently large $n$, where $\varepsilon>0$ may be chosen arbitrarily.

The construction increases both the computational and communication complexities to be the sum of the previous computational and communication complexities. Therefore, the new bound for both will be the old computational bound.

Because of space limitations, the proof of Theorem 1 on the preceding page is given in the full version (on ePrint) [12.

In the full version we also prove Corollary 1, which is a reduction in the complexity in some cases, namely when the depth is large and when $X$ (the maximal number of connections from one layer to others) is large.

Corollary 1. With the modification of the full version, the complexities of Theorem 1 can be altered to

$$
\mathcal{O}(\log \operatorname{depth}(C) \log n \log |C| \cdot|C|)+\operatorname{poly}(n, \log |C|) \cdot \operatorname{depth}(C) \log \operatorname{depth}(C)
$$

for communication and

$$
\mathcal{O}\left(\log \operatorname{depth}(C) \log ^{2} n \log |C| \cdot|C|\right)+\operatorname{poly}(n, \log |C|) \cdot \operatorname{depth}(C) \log \operatorname{depth}(C)
$$

for computation.

\section{Application to Two-Party Cryptography}

In this section we sketch the application of our main result to reducing the computational overhead of zero-knowledge proofs and secure two-party computation. 
In [20] it is shown how to obtain a zero-knowledge proof for the satisfiability of a circuit $C$ from any MPC protocol for $n$ servers in which one client ("the prover") has an input $w$ and another client ("the verifier") should output $C^{\prime}(w)$, where $C^{\prime}$ is a constant-depth circuit of roughly the same size as $C$ which is easily determined by $C$. If the MPC protocol is adaptively secure against an active adversary who corrupts the prover and a constant fraction of the servers, the resulting zero-knowledge protocol will have soundness error of $2^{-\Omega(n)}$ plus the correctness error of the MPC protocol. The simulation error corresponds to that of the MPC protocol. The efficiency of the zero-knowledge protocol is essentially the same as that of the MPC protocol, excluding the cost of $n$ commitments to strings whose total size is roughly the communication complexity of the MPC protocol.

The above transformation was combined with the MPC techniques from [119 to yield zero-knowledge proofs with a constant communication overhead. However, to guarantee soundness error of $2^{-k}$, the computational overhead of this protocol must be $\Omega(k)$, even if ideal commitments are used. Plugging in our main result, we obtain a perfect zero-knowledge protocol in the commitmenthybrid model (i.e., using ideal commitments) in which both the communication and computation overhead are polylogarithmic in $k$. As a side benefit, the perfect security of our protocol allows for a simpler and more round-efficient transformation into a zero-knowledge proof protocol (see [20], Section 4).

To implement the commitment-hybrid model, we can use the constant overhead constructions from [21] or the polylog-overhead constructions from [1]. The latter have the advantage of relying on fairly standard cryptographic assumptions, related to the intractability of decoding random linear codes or learning with errors.

We note that in the case of zero-knowledge arguments (with computational soundness), it is possible to combine the PCP-based approach of [23 25] for efficient arguments with state of the art PCP constructions 4 and efficient lattice-based constructions of collision-resistant hash functions [26]24 to get alternative constructions with polylogarithmic computational overhead. However, other than offering only computational soundness, the resulting protocol requires stronger assumptions, inherits the complex and seemingly impractical nature of current PCP constructions, and does not allow to eliminate the need for cryptography using preprocessing.

We finally note that similar results can be obtained in the more general context of secure two-party computation. One approach to obtain these results is to apply the GMW-compiler [17], with the efficient zero-knowledge proofs described above, to a constant-overhead protocol for the semi-honest model from [21]. The latter protocol relies on the existence of a pseudorandom generator stretching $n$ bits to $n^{2}$ bits in which each bit of the output depends on just a constant number of input bits - a plausible but nonstandard assumption. Another approach, which can offers unconditional security in the OT-hybrid model, is to instantiate the protocol compiler from [22] with our main protocol as the "outer protocol". 


\section{On the Relevance of Gentry's Scheme}

The recent breakthrough of Gentry [16, suggesting the first plausible candidate for a fully homomorphic encryption scheme, has a great impact on the theoretical efficiency of MPC. By distributing the key generation and decryption of Gentry's scheme between the $n$ players, it is possible to obtain general constant-round MPC protocols whose communication complexity only depends on $n$ and the length of the inputs and outputs of $C$ rather than the size of $C$. We note, however, that this protocol can only provide computational security (under a non-standard assumption) and, perhaps more importantly, its computational overhead involves a large polynomial in the security parameter. The high computational cost seems to make Gentry's scheme, in its current form, too inefficient for practical purposes. Finally, for circuits whose output length is not much smaller than their size (as in the case of performing a large number of simple computations), even the communication overhead of this protocol becomes a large polynomial in $k$ and $n$. In contrast, our protocol has the same overhead even in this case. In light of the above, it seems fair to conclude that Gentry's result has limited relevance to the results of the present work from both a theoretical and from a practical point of view.

\section{References}

1. Applebaum, B., Cash, D., Peikert, C., Sahai, A.: Fast cryptographic primitives and circular-secure encryption based on hard learning problems. In: Halevi, S. (ed.) CRYPTO 2009. LNCS, vol. 5677, pp. 595-618. Springer, Heidelberg (2009)

2. Beerliová-Trubíniová, Z., Hirt, M.: Perfectly-secure MPC with linear communication complexity. In: Canetti, R. (ed.) TCC 2008. LNCS, vol. 4948, pp. 213-230. Springer, Heidelberg (2008)

3. Ben-Or, M., Goldwasser, S., Wigderson, A.: Completeness theorems for noncryptographic fault-tolerant distributed computation (extended abstract). In: STOC, pp. 1-10. ACM, New York (1988)

4. Ben-Sasson, E., Goldreich, O., Harsha, P., Sudan, M., Vadhan, S.P.: Short pcps verifiable in polylogarithmic time. In: IEEE Conference on Computational Complexity, pp. 120-134. IEEE Computer Society, Los Alamitos (2005)

5. Benes, V.E.: Optimal rearrangable multistage connecting networks. The Bell System Technical Journal 43, 1641-1656 (1964)

6. Bracha, G.: An $\mathrm{O}(\log \mathrm{n})$ expected rounds randomized byzantine generals protocol. J. ACM 34(4), 910-920 (1987)

7. Canetti, R.: Universally composable security: A new paradigm for cryptographic protocols. In: FOCS 2001: Proceedings of the 42nd IEEE symposium on Foundations of Computer Science, Washington, DC, USA, pp. 136-145. IEEE Computer Society, Los Alamitos (2001)

8. Chaum, D., Crépeau, C., Damgard, I.: Multiparty unconditionally secure protocols. In: STOC 1988: Proceedings of the twentieth annual ACM symposium on Theory of computing, pp. 11-19. ACM, New York (1988) 
9. Chen, H., Cramer, R.: Algebraic geometric secret sharing schemes and secure multiparty computations over small fields. In: Dwork, C. (ed.) CRYPTO 2006. LNCS, vol. 4117, pp. 521-536. Springer, Heidelberg (2006)

10. Cramer, R., Damgård, I., Nielsen, J.B.: Multiparty computation from threshold homomorphic encryption. In: Pfitzmann, B. (ed.) EUROCRYPT 2001. LNCS, vol. 2045, pp. 280-299. Springer, Heidelberg (2001)

11. Damgård, I., Ishai, Y.: Scalable secure multiparty computation. In: Dwork, C. (ed.) CRYPTO 2006. LNCS, vol. 4117, pp. 501-520. Springer, Heidelberg (2006)

12. Damgård, I., Ishai, Y., Krøigaard, M.: Perfectly secure multiparty computation and the computational overhead of cryptography. Cryptology ePrint archive, report 2010/131 (2010), http://eprint.iacr.org/

13. Damgård, I., Ishai, Y., Krøigaard, M., Nielsen, J.B., Smith, A.: Scalable multiparty computation with nearly optimal work and resilience. In: Wagner, D. (ed.) CRYPTO 2008. LNCS, vol. 5157, pp. 241-261. Springer, Heidelberg (2008)

14. Franklin, M.K., Yung, M.: Communication complexity of secure computation (extended abstract). In: STOC, pp. 699-710. ACM, New York (1992)

15. Gennaro, R., Rabin, M.O., Rabin, T.: Simplified VSS and fast-track multiparty computations with applications to threshold cryptography. In: PODC 1998: Proceedings of the seventeenth annual ACM symposium on Principles of distributed computing, pp. 101-111. ACM, New York (1998)

16. Gentry, C.: Fully homomorphic encryption using ideal lattices. In: STOC 1909: Proceedings of the 41st annual ACM symposium on Theory of computing, pp. 169-178. ACM, New York (2009)

17. Goldreich, O., Micali, S., Wigderson, A.: How to play any mental game or a completeness theorem for protocols with honest majority. In: STOC, pp. 218-229. ACM, New York (1987)

18. Hirt, M., Maurer, U.M.: Player simulation and general adversary structures in perfect multiparty computation. J. Cryptology 13(1), 31-60 (2000)

19. Hirt, M., Maurer, U.M., Przydatek, B.: Efficient secure multi-party computation. In: ASIACRYPT 2000: Proceedings of the 6th International Conference on the Theory and Application of Cryptology and Information Security, London, UK, pp. 143-161. Springer, Heidelberg (2000)

20. Ishai, Y., Kushilevitz, E., Ostrovsky, R., Sahai, A.: Zero-knowledge from secure multiparty computation. In: STOC 2007: Proceedings of the thirty-ninth annual ACM symposium on Theory of computing, pp. 21-30. ACM, New York (2007)

21. Ishai, Y., Kushilevitz, E., Ostrovsky, R., Sahai, A.: Cryptography with constant computational overhead. In: STOC 2008: Proceedings of the 40th annual ACM symposium on Theory of computing, pp. 433-442. ACM, New York (2008)

22. Ishai, Y., Prabhakaran, M., Sahai, A.: Founding cryptography on oblivious transfer — efficiently. In: Wagner, D. (ed.) CRYPTO 2008. LNCS, vol. 5157, pp. 572-591. Springer, Heidelberg (2008)

23. Kilian, J.: A note on efficient zero-knowledge proofs and arguments (extended abstract). In: STOC 1992: Proceedings of the twenty-fourth annual ACM symposium on Theory of computing, pp. 723-732. ACM, New York (1992)

24. Lyubashevsky, V., Micciancio, D.: Generalized compact knapsacks are collision resistant. In: Bugliesi, M., Preneel, B., Sassone, V., Wegener, I. (eds.) ICALP 2006, Part II. LNCS, vol. 4052, pp. 144-155. Springer, Heidelberg (2006)

25. Micali, S.: Computationally sound proofs. SIAM J. Comput. 30(4), 1253-1298 (2000) 
26. Peikert, C., Rosen, A.: Efficient collision-resistant hashing from worst-case assumptions on cyclic lattices. In: Halevi, S., Rabin, T. (eds.) TCC 2006. LNCS, vol. 3876, pp. 145-166. Springer, Heidelberg (2006)

27. Rabin, T., Ben-Or, M.: Verifiable secret sharing and multiparty protocols with honest majority. In: STOC 1989: Proceedings of the twenty-first annual ACM symposium on Theory of computing, pp. 73-85. ACM, New York (1989)

28. Shamir, A.: How to share a secret. Commun. ACM 22(11), 612-613 (1979)

29. Waksman, A.: A permutation network. J. ACM 15(1), 159-163 (1968) 